\title{
Statin therapy modulates thickness and inflammatory profile of human epicardial adipose tissue
}

\author{
Valentina Parisi ${ }^{\mathrm{a}, *, 1}$, Laura Petraglia ${ }^{\mathrm{a}, 1}$, Vittoria D'Esposito ${ }^{\mathrm{a}, 1}$, Serena Cabaro ${ }^{\mathrm{a}, 1}$, Giuseppe Rengo ${ }^{\mathrm{a}, 1}$, \\ Aurelio Caruso $^{\mathrm{b}, 1}$, Maria Gabriella Grimaldi ${ }^{\mathrm{b}, 1}$, Francesco Baldascino ${ }^{\mathrm{b}, 1}$, Antonio De Bellis ${ }^{\mathrm{b}, 1}$, Dino Vitale ${ }^{\mathrm{b}, 1}$, \\ Roberto Formisano a,1, Adele Ferro c ${ }^{\mathrm{c}}$, Stefania Paolillo d,1, Laurent Davin e,1, Patrizio Lancellotti e,f,1, \\ Pietro Formisano ${ }^{\mathrm{a}, 1}$, Pasquale Perrone Filardi ${ }^{\mathrm{g}, 1}$, Nicola Ferrara ${ }^{\mathrm{a}, 1}$, Dario Leosco ${ }^{\mathrm{a}, 1}$ \\ a Department of Translational Medical Science, University of Naples Federico II, Italy \\ b Casa di Cura San Michele, Maddaloni, CE, Italy \\ ${ }^{\mathrm{c}}$ Institute of Biostructure and Bioimaging, CNR, Naples \\ d Italy SDN Foundation, Institute of Diagnostic and Nuclear Development, Naples, Italy \\ e University of Liège Hospital, GIGA Cardiovascular Sciences, Departments of Cardiology, Heart Valve Clinic, CHU Sart Tilman, Liège, Belgium \\ ${ }^{\mathrm{f}}$ Gruppo Villa Maria Care and Research, Anthea Hospital, Bari, Italy \\ ${ }^{g}$ Department of Advanced Biomedical Science, University Federico II of Naples, Naples, Italy
}

\section{A R T I C L E I N F O}

\section{Article history:}

Received 14 May 2018

Received in revised form 12 June 2018

Accepted 27 June 2018

Available online 28 June 2018

\section{Keywords:}

Epicardial adipose tissue

Statin

Inflammation

Aortic stenosis

\begin{abstract}
A B S T R A C T
Background: Epicardial adipose tissue (EAT) thickness and pro-inflammatory status has been shown to be associated with several cardiac diseases, including aortic stenosis (AS). Thus, cardiac visceral fat could represent a potential new target for drugs. In the present study we evaluate the effect of statin therapy on EAT accumulation and inflammation.

Methods: Echocardiographic EAT thickness was assessed in 193 AS patients taking (n.87) and not taking (n.106) statins, undergoing cardiac surgery. To explore the association between statin therapy and EAT inflammation, EAT biopsies were obtained for cytokines immunoassay determination in EAT secretomes. An in vitro study was also conducted and the modulation of EAT and subcutaneous adipose tissue (SCAT) secretomes by atorvastatin was assessed in paired biopsies.

Results: Statin therapy was significantly associated with lower EAT thickness $(\mathrm{p}<0.0001)$ and with lower levels of EAT-secreted inflammatory mediators $(p<0.0001)$. Of note, there was a significant correlation between EAT thickness and its pro-inflammatory status. In vitro, atorvastatin showed a direct anti-inflammatory effect on EAT which was significantly higher compared to the SCAT response to statin incubation $(\mathrm{p}<0.0001)$.

Conclusions: The present study indicates a robust association between statin therapy and reduced EAT accumulation in patients with AS. The present data also suggest a direct relationship between EAT thickness and its inflammatory status, both modulated by statin therapy. The in vitro results support the hypothesis of a direct action of statins on EAT secretory profile. Overall our data suggest EAT as a potential new therapeutic target for statin therapy.
\end{abstract}

(c) 2018 Elsevier B.V. All rights reserved.

\section{Introduction}

Statin therapy has an established role on primary and secondary prevention of cardiovascular diseases [1-5]. The beneficial effects of this class of drugs on cardiovascular outcomes go beyond the lipid levels control and accumulating evidence support the relevance of statin-related pleiotropic activities. Beside the inhibition of HMG-CoA

\footnotetext{
* Corresponding author at: Department of Translational Medical Sciences, University 'Federico II', Via S. Pansini 5, 80131 Naples, Italy.

E-mail address: valentina.parisi@unina.it (V. Parisi).

1 This author takes responsibility for all aspects of the reliability and freedom from bias of the data presented and their discussed interpretation.
}

reductase and mevalonate pathway, statins may affect a variety of processes such as cell signalling, cell differentiation and proliferation [6]. At this regard, it has been emphaticized the role of statins in attenuating inflammation, a well-recognized substrate of the atherosclerotic process [7-9]. Few data also suggest a potential anti-inflammatory effect of statins on visceral fat depots, such as omental and thymus adipose tissue $[10,11]$, however no data are available on cardiac visceral fat. Epicardial adipose tissue (EAT), the visceral fat of the heart, represents a source of several adipocytokines and other bioactive molecules $[12,13]$. Because of its proximity to the myocardium and absence of fascial boundaries, EAT directly influences myocardial homeostasis through vasocrine and paracrine mechanisms. Increased secretion of EAT inflammatory mediators are associated to pathological cardiac 
conditions, including coronary atherosclerosis and aortic stenosis $[12,14]$. The potential mechanistic involvement of EAT in the development and progression of cardiac diseases gave raised to hypothesize that the cardiac visceral fat could represent a new potential therapeutic target other than a risk factor for cardiac pathologies. However, until now, definitive evidence of the efficacy of specific drug therapies in modifying EAT pathologic phenotype are not still available. To this aim, in the present study, we want to explore, in vivo and in vitro, whether statin therapy could regulate EAT accumulation and proinflammatory activity in patients with severe calcific aortic stenosis (AS), whose association with increased EAT thickness and inflammatory status has been recently reported by our group [14].

\section{Methods}

\subsection{Study population}

From April 2014 to December 2017, we enrolled 193 patients with isolated severe calcific aortic stenosis admitted for clinical evaluation prior aortic valve replacement to two cardiac surgery centers (University of Naples Federico II, Italy, and San Michele Hospital, Maddaloni, CE, Italy). Of these, 87 patients (45.1\%) were on statin therapy for a duration ranged from 3 to 72 months. Since the low proportion of non-statin users in patients with coronary artery disease (CAD), we excluded from the study subjects with previous diagnosis of CAD and with significant CAD at the pre-operative coronary angiography. Furthermore, in order to minimize potential confounding factors, we also excluded patients with chronic inflammatory diseases and cancer since the association of these pathologic conditions with visceral fat inflammation. From all patients we collected demographic and clinical data. The study protocol conforms to the ethica guidelines of the 1975 Declaration of Helsinki. All the study procedures received approva by our institution's human research committee (Protocol n. 173/13). All patients provided written informed consent before their inclusion into the study.

\subsection{Echocardiography}

All patients underwent complete echocardiographic study (GE vivid E9) and the diagnosis of severe aortic stenosis was confirmed in accordance to the recently published criteria [15]. The maximum EAT thickness was measured in systole, from the parasternal long axis view that visualizes EAT tissue between the right ventricle and the ascending aorta. Measurements of EAT thickness were performed offline by two independent, blinded, echocardiographers. The average value from three cardiac cycles was used for the statistical analysis.

\subsection{Tissues collection}

Throughout the entire enrollment period, we collected EAT biopsies from a subgroup of 31 patients undergoing cardiac surgery for valve replacement. EAT biopsy samples (average 0.1 to $0.5 \mathrm{~g}$ ) were taken near the proximal right coronary artery before the initiation of cardiopulmonary bypass. For the in vitro study also subcutaneous adipose (SCAT) (average 0.1 to $0.5 \mathrm{~g}$ ) samples were obtained from the chest.

\subsection{Cytokine and growth factor assay}

From EAT biopsies, secretomes were obtained as follows: tissues were weighted, cut into small pieces, and transferred into a 12-well plate. According to tissue weight, serum-free DMEM ( $1 \mathrm{~mL}$ medium/ $0.1 \mathrm{~g}$ tissue) was added to the well and incubated at $37{ }^{\circ} \mathrm{C}$ in a $\mathrm{CO}_{2}$ incubator. After $24 \mathrm{~h}$, medium was collected and centrifuged at $14,000 \mathrm{~g}$ to remove debris and analysed for cytokines and growth factors content, as described below.

EAT conditioned media was screened for the concentration of Interleukin (IL)-1B, IL-1ra, IL-2, IL-4, IL-5, IL-6, IL-7, IL-8, IL-9, IL-10, IL-12, IL-13, IL-15, IL-17, basic Fibroblast Growth Factor (FGF), Eotaxin, Granulocyte-Colony Stimulating Factor (G-CSF), Granulocyte-Macrophage Colony Stimulating Factor (GM-CSF), Interferon (IFN)- $\gamma$, Interferon gamma-Induced Protein (IP)-10, Monocyte Chemoattractant Protein (MCP)-1, Macrophage Inflammatory Protein 1-alpha/beta (MIP-1 $\alpha$, MIP-1 $\beta$ ), Platelet-Derived Growth Factor (PDGF), Regulated on Activation Normal T-cell Expressed and Secreted RANTES/CCL5, Tumor Necrosis Factor (TNF)- $\alpha$, and Vascular Endothelial Growth Factor (VEGF), using the Bio-Plex multiplex Human Cytokine and Growth factor kits (Bio-Rad) according to the manufacturer's protocol.

\subsection{In vitro study}

To verify whether statins might directly affect EAT inflammatory profile, we in vitro treated with atorvastatin EAT and SCAT specimens obtained from patients not taking statins. To reduce any confounding factor, we used paired biopsies. In particular, each biopsy was cut in two equal pieces, transferred into culture plates and treated or not with $2 \mu \mathrm{M}$ Atorvastatin at $37{ }^{\circ} \mathrm{C}$ in a $\mathrm{CO}_{2}$ incubator. After $24 \mathrm{~h}$, conditioned media were collected, centrifuged at 14,000g to remove debris and analysed for cytokines and growth factors content, as described above.

\subsection{Materials}

Media were from Lonza (Lonza Group Ltd., Basel, Switzerland). Atorvastatin was from Sigma-Aldrich (St. Louis, MO, USA)

\subsection{Statistical analysis}

In order to assess the association between statin therapy and EAT thickness a multivariable regression model was tested between the echocardiographic EAT thickness, as dependent variable, whereas statin use, acetyl salicylic acid use (ASA), age, male sex, body mass index (BMI), hypertension, diabetes, dyslipidaemia and smoking habit were included as independent variables. Statin therapy was tested as effect modifier while the others variables were tested as possible confounders and were selected from factors potentially associated with EAT thickness. The model building procedure was centred on the multivariable fractional polynomial (MFP) algorithm [16] that allows both backward stepwise variable selection and assessment of the functional form (linearity/non linearity) on the continuous variables. The possible presence of multicollinearity between the independent variables tested in the model was checked by measuring the variance inflation factor (VIF). The relevance of the factors included in the final model was assessed by each partial contribution to the global explained variance (R2) that was accomplished by the Shapley-Owen decomposition algorithm [17]. The stability of the results obtained in the final model was assessed by measuring the internal validity with nonparametric bootstrap sampling [18]. Briefly, given the parameters of the final model obtained with the mentioned model-building procedure, the stability of each factor tested in the model was measured by the frequency that this factor is selected as 'significant' in a large (1000) number of bootstrap replications of the dataset (BIF).

In order to verify in vivo the relationship between EAT cytokine levels and statin therapy, a two-way ANOVA full model with interaction was used on logarithm of cytokine concentration with statin use and cytokine type as main effects. A two-way ANOVA full model with cytokine type and specimen site (EAT or SCAT) was used to investigate in vitro differences of cytokine response to atorvastatin stimulation between EAT and SCAT specimens.

\section{Results}

\subsection{Patient characteristics}

Table 1 illustrates demographic and clinical characteristics of the overall study population. The mean age was $72.2 \pm 10.5$ years and $43 \%$ of patients were males. As regard to common atherosclerotic risk factors, $75.6 \%$ of patients were hypertensives, $29.5 \%$ were diabetics, $32.1 \%$ were smokers and $53.9 \%$ were dyslipidemics. Seventy percent of patients assumed ACE-inhibitors/sartans, 24.9\% calcium-channel blockers, $64.8 \%$ beta-blockers, and $60 \%$ aspirin. Use of statins was reported in $45 \%$ of patients. Eighty-two percent of statin treated patients were on atorvastatin ( $40 \mathrm{mg}$ once daily, mean duration $=24 \pm$ 17 months), $11 \%$ on rosuvastatin (20 mg once daily, mean duration = $19 \pm 12$ months), and $7 \%$ on pravastatin (20 mg once daily, mean duration $=31 \pm 18$ months). At echocardiography, all patients fulfilled criteria of severe AS [15], mean left ventricular ejection fraction (LVEF) was $66.0 \pm 10.8 \%$, thus indicating a globally preserved LV systolic function. Mean EAT thickness was $11.3 \pm 3.2 \mathrm{~mm}$. Intra and interobserver reproducibility for echocardiographic EAT thickness assessment was excellent ( 0.964 and 0.953 , respectively).

\subsection{Multivariable determinants of EAT thickness}

Table 2 reports the multivariable determinants of EAT thickness. Multicollinearity check gave a mean VIF equal to 1.2 with an observed maximum of 1.3 , well below the critical values of $4[19,20]$, thus documenting absence of multicollinearity bias. Statin therapy and dyslipidaemia were the only two factors retained significant in the final model. A $16 \%$ fraction of the total variance observed in the EAT thickness was explained by the model. Notably, $>96 \%$ of this was accounted by the statin therapy while $<4 \%$ was attributable to the presence of dyslipidaemia. Both statin use and dyslipidaemia showed a linear functional form in all bootstrap resamples. Interestingly, statin therapy showed an excellent significance stability (BIF $=100 \%$ ) when compared to dyslipidaemia that resulted significant only in the $55 \%$ of 
Table 1

Demographic and clinical characteristics of study population.

\begin{tabular}{ll}
\hline Age, mean \pm SD & $72.2 \pm 10.5$ \\
\hline Gender, male \% (n) & $43.0 \%(83)$ \\
BMI, mean \pm SD & $28.4 \pm 5.4$ \\
Hypertension, \% (n) & $75.6 \%(146)$ \\
Diabetes, \% (n) & $29.5 \%(57)$ \\
Smokers, \% (n) & $32.1 \%(62)$ \\
Dyslipidemia, \% (n) & $53.9 \%(104)$ \\
ACE-I/ARB \% (n) & $66.8 \%(129)$ \\
CCBs, \% (n) & $24.9 \%(48)$ \\
BBs, \% (n) & $64.8 \%(125)$ \\
Statins, \% (n) & $45.1 \%(87)$ \\
ASA, \% (n) & $59.6 \%(115)$ \\
LVEF, \% & $66.0 \pm 10.8$ \\
EAT thickness, mm & $11.3 \pm 3.2$ \\
\hline
\end{tabular}

$\mathrm{BMI}=$ body mass index; $\mathrm{ACE}-\mathrm{I}=\mathrm{ACE}$ inhibitors; $\mathrm{ARBs}=$ angiotensin receptor blockers; $\mathrm{CCBs}=$ calcium channel blockers; $\mathrm{BBs}=$ beta-blockers; ASA = Acetylsalicylic Acid; LVEF = left ventricular ejection fraction; EAT $=$ epicardial adipose tissue.

the resampled datasets. Although the potential anti-inflammatory effects of statin and ASA use on EAT thickness, no significant interaction was found between these two variables in the model.

\subsection{In vivo effects of statin therapy on EAT inflammatory profile}

We explored the relationship between statin therapy and the concentration of cytokines in EAT secretome. The two-way ANOVA (Table 3, Supplemental material) showed a significant association between statin therapy and EAT cytokines concentrations. This effect is also visually appreciable in the Fig. 1 showing the cytokines that were particularly affected by statin therapy. The not significant cytokine/statin interaction leads to conclude that such association is not modulated by cytokine types. Probably, a small difference between cytokine types compared to data variability hides the interaction effect. Of interest, cytokine concentrations were significantly ( $\mathrm{x} \leq 0.001)$ and directly correlated with EAT thickness at a two-way ANOVA that took into account cytokine type (Table 4, Supplemental material). Moreover, even in this subgroup of patients, the significant, direct relationship between EAT thickness and statin use was confirmed ( $p \leq 0.001$ ), thus supporting the hypothesis of a close interaction between EAT accumulation and its exalted inflammatory profile.

\subsection{In vitro effects of atorvastatin on EAT inflammatory profile}

To confirm the statin related effect on EAT and minimize any possible confounding effect, we planned an in vitro experiment with paired biopsies matching the effect of statin on EAT and SCAT. To compare the effect of statin stimulation, the results of the 27 cytokines analysis were expressed as the concentration ratios between the treated/not treated specimen halves. The two-way ANOVA analysis

\section{EAT secretome cytokines}

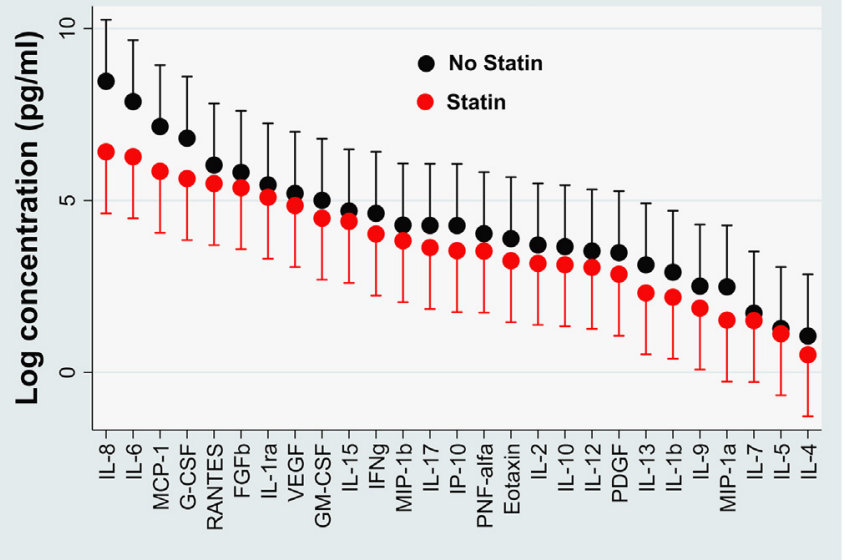

Fig. 1. EAT cytokine secretome concentrations \pm mean square error in patients taking or not statin therapy. EAT; epicardial adipose tissue.

(Table 5, supplemental material) showed a significant lower ratio in the EAT specimen, denoting a greater reduction of cytokines concentration in the EAT tissue after statin incubation when compared to SCAT incubation response. The significant interaction with cytokine type indicates that cytokine type modulates this effect and gives rise to a differentiated response for each cytokine, as evidenced in Fig. 2 were each cytokine ratio was ordered by the response magnitude.

\section{Discussion}

In a model of atherosclerotic cardiac disease, the calcific AS, whose association with increased EAT thickness and inflammatory status has been recently reported [14], we explored, in vivo and in vitro, whether statin therapy might affect EAT accumulation and inflammatory profile. Major findings of the present study are: 1) statin therapy is significantly associated to a reduced EAT thickness; 2 ) the association between statin therapy and reduction of EAT accumulation is paralleled by an attenuation of EAT inflammatory profile; 3 ) as indicated by the vitro studies, statin have a direct and selective anti-inflammatory effect on EAT.

EAT represents a marker of visceral adiposity and its mass is increased in several metabolic and cardiovascular diseases, such as metabolic syndrome, insulin resistance and CAD [21-24]. We have previously reported [14] that EAT thickness is also increased in patients with AS and represents a relevant source of inflammatory cytokines. In the present study, in order to explore a potential effect of statins on EAT pro-inflammatory profile, we selected a population of patients with AS, since, in this clinical setting, statin prescription is not mandatory following the recommendations of the current guidelines. This methodological strategy allowed us to have a control group of patients

Table 2

Multivariable regression analysis of variables associated with EAT thickness.

\begin{tabular}{|c|c|c|c|c|c|}
\hline & \multicolumn{5}{|c|}{ Global R2 $=0.16$} \\
\hline & Coeff. & $\mathrm{p}$ & $\begin{array}{l}\text { Percent fraction } \\
\text { of global R2 (\%) }\end{array}$ & $\begin{array}{l}\text { Bootstrap Inclusion } \\
\text { Frequency (\%) }\end{array}$ & $\begin{array}{l}\text { Linearity Stability } \\
(\%)\end{array}$ \\
\hline Age & 0.0 & 0.17 & NA & $32.0 \%$ & NA \\
\hline Gender (male) & -0.7 & 0.13 & NA & $24.2 \%$ & NA \\
\hline BMI & 0.1 & 0.09 & NA & $46.7 \%$ & NA \\
\hline Hypertension & 0.4 & 0.4 & NA & $5.7 \%$ & NA \\
\hline Diabetes & -0.1 & 0.84 & NA & $8.0 \%$ & NA \\
\hline Dyslipidemia & 1.0 & 0.04 & $3.7 \%$ & $63.9 \%$ & $100.0 \%$ \\
\hline Smoking habit & 0.07 & 0.87 & NA & $15.0 \%$ & NA \\
\hline Statin therapy & -2.8 & $\leq 0.0001$ & $96.3 \%$ & $100.0 \%$ & $100.0 \%$ \\
\hline ASA & 0.33 & 0.48 & NA & $14.6 \%$ & NA \\
\hline
\end{tabular}

ASA = acetylsalicylic acid; BMI = body mass index; EAT = epicardial adipose tissue; NA not applicable. 


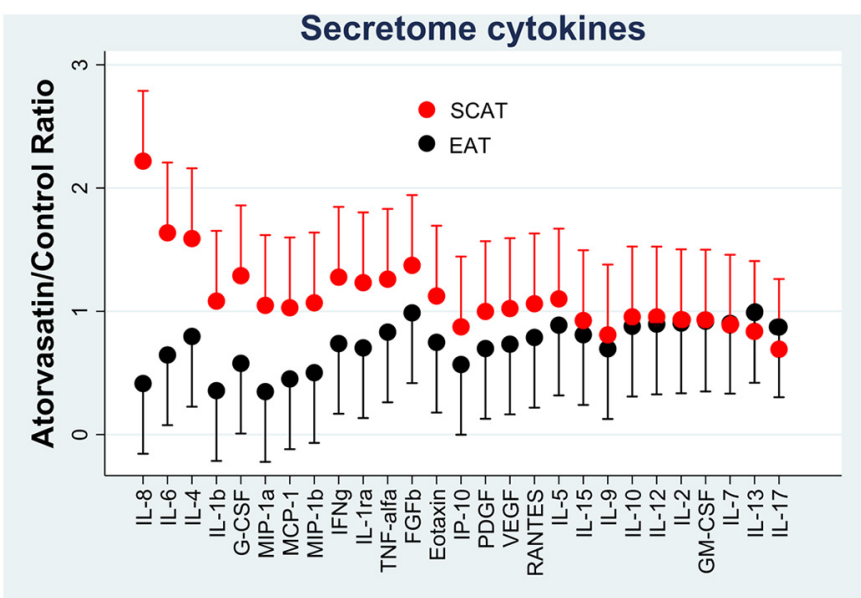

Fig. 2. In vitro atorvastatin/control ratios of cytokine secretome concentrations in EAT and SCAT. EAT; epicardial adipose tissue. SCAT; Subcutaneous adipose tissue.

not treated with statins. In this population, we observed that statin therapy was a significant predictor of EAT thickness, when added to a clinical model including age, male sex, BMI, hypertension, diabetes, dyslipidaemia and smoking habit. Of note, the linear functional form in all bootstrap resamples and the excellent significance stability of this association strengthened the result of the performed analysis. This finding is in line with previous evidence obtained in other settings [25-27] indicating a statin-related reduction of EAT in patients with CAD, atrial fibrillation, and dyslipidaemia. Interestingly, our results suggest, for the first time, that the association of statin therapy with a lower EAT thickness is paralleled by an attenuation of the inflammatory profile of the cardiac visceral fat. In particular, we observed a significant association between statin therapy, EAT thickness and levels of cytokines secreted from this tissue.

A direct evidence of the modulating activity of statin therapy on EAT pro-inflammatory profile was provided by the in vitro results of the present study. Analysing EAT paired biopsies obtained from AS patients not assuming statins, atorvastatin showed a significant effect in reducing cytokine concentrations in cardiac visceral fat. These observations are corroborated by previous in vitro animal studies describing the anti-inflammatory effect of statins on visceral adipose tissue. In this regard, statins have been shown to suppress macrophage-mediated inflammatory response of the adipocytes extracted from the visceral fat of obese mice [28]. Overall, these evidence support the role of statins in regulating the secretory properties of visceral fat and our study now extends these previous observations to the cardiac human visceral fat. Noteworthy, our data also indicated that the anti-inflammatory effect of atorvastatin was significantly greater in EAT than in SCAT. The different responses of these two adipose tissues to statin stimulation represent another evidence that EAT and SCAT not only differ in terms of morphologic characteristics but also show a different secretory phenotype. This confers a unique role to EAT in cardiac diseases and strengthens the concept that cardiac visceral fat is a more metabolically active tissue than SCAT $[11,14]$.

A significant interaction with cytokine type was revealed by in vitro experiments of the present study, indicating that cytokine type modulated the response of EAT to atorvastatin. Of interest, this effect seemed to be mainly driven by IL-6 and IL-8 (Fig. 2), two of the most studied cytokines in the atherosclerotic process and already associated to the statin related benefit in experiments conducted on coronary artery endothelial cells $[29,30]$. A similar trend for statin induced IL-6 and Il-8 reduction, although not statistically significant, was found in vivo.

In conclusion, the present study suggests, for the first time, an association between statin therapy, EAT thickness and inflammatory profile in patients with AS. In particular, our results indicate that the statin related EAT anti-inflammatory effect is the result of a direct action on cardiac visceral fat and is paralleled by a reduction of EAT thickness at echocardiography. Although our results may give only preliminary information on statin doses in AS patients, they encourage the realization of future studies in this field that could be helpful to cardiologists to define the optimal doses for the treatment of this patients' population. Finally, since the relationship between EAT thickness and its secretory profile, other studies should be planned to evaluate if the echocardiographic evaluation of this cardiac adipose deposit could be utilized as a surrogate marker of the EAT inflammatory status in the clinical practice and whether the statin-related effect on EAT morphological and functional remodelling could impact on cardiovascular outcomes.

\subsection{Study limitations}

This was not a longitudinal study, thus, EAT thickness was not monitored over time before and during statin therapy. However, in the present study, we needed to enroll patients with advanced aortic valve disease. All these patients underwent urgent surgical valve replacement, thus, giving us the opportunity, in a subgroup of them, to obtain human EAT tissues for in vivo and in vitro experiments. Future longitudinal studies, conducted on patients with less advanced AS, are necessary to follow over time the effect of statin therapy on EAT thickness.

Since the limited availability of human EAT biopsies, the in vitro section of the present study evaluated the anti-inflammatory effects of a single statin, atorvastatin, thus avoiding us to extend this observation to other components of this class of drugs. The choice of atorvastatin was based on the following reasons: 1) the majority of our patients on statin regimen were on atorvastatin therapy; 2) previous evidence have already demonstrated an anti-inflammatory effect of atorvastatin on human adipocytes in other settings [13]. Further studies are needed to explore whether the observed anti-inflammatory effects on EAT might be obtained with other molecules.

Both in vivo and in vitro studies on EAT secretome have been conducted on the whole tissue. Since the cell type mix in EAT, we cannot say whether the anti-inflammatory effect of statin has to be mainly referred to a direct activity on the adipocyte secretory properties or on the inflammatory cell lines expressed in this tissue.

It would have been more reliable once a positive control was included in the in vitro study but the limited amount of EAT tissues was not sufficient to perform this kind of experiments.

An apparent limitation of this study is represented by the lack of data regarding systemic inflammation in our AS population. In this regard, our group and other have already demonstrated that local inflammation assessed at EAT level is independent from the systemic levels of the explored cytokines [12,14]. As regard the systemic effect of statin therapy in the AS population, previous studies have demonstrated that statins induce reduction of systemic inflammation, assessed by circulating C-reactive protein (CRP) levels [31]. However, CRP is predominantly produced in the liver, thus being poor affected by localized inflammation phenomena, such as that explored in the present study [32].

Echocardiographic EAT measurement has several advantages, such as low cost and easy availability, but it also has some limitations because it might not fully reflect the variability of fat thickness or total EAT volume as assessed with cardiac magnetic resonance. However, the EAT echocardiographic evaluation has been reported to correlate with magnetic resonance measurements [33] and has an excellent reproducibility [34], as also confirmed in the current study.

\section{Conflicts of interest}

None. 


\section{Appendix A. Supplementary data}

Supplementary data to this article can be found online at https://doi. org/10.1016/j.ijcard.2018.06.106.

\section{References}

[1] Randomized trial of cholesterol lowering in 4444 patients with coronary heart disease: the Scandinavian Simvastatin Survival Study (4S), Lancet 334 (1994) 1383-1389.

[2] F.M. Sacks, M.A. Pfeffer, L.A. Moye, The effect of pravastatin on coronary events after myocardial infarction in patients with average cholesterol levels, N. Engl. J. Med. 335 (1996) 1001-1009.

[3] Prevention of cardiovascular events and death with pravastatin in patients with coronary heart disease and broad range of initial cholesterol levels. The long-term intervention with pravastatin in Ischaemic disease (LIPID) study group, N. Engl. J. Med. 339 (1998) 1349-1357.

[4] West of Scotland coronary prevention study: identification of high-risk groups and comparison with other cardiovascular intervention trials, Lancet 346 (1996) 1339-1342.

[5] J.R. Downs, M. Clearfield, S. Weis, Primary prevention of acute coronary events with lovastatin in men and women with average cholesterol levels, N. Engl. J. Med. 339 (1999) 1615-1622.

[6] A. Corsini, S. Bellosta, R. Baetta, R. Fumagalli, R. Paoletti, F. Bernini, New insights into the pharmacodynamic and pharmacokinetic properties of statins, Pharmacol. Ther. 84 (1999) 413-428.

[7] V.Z. Rocha, P. Libby, Obesity, inflammation, and atherosclerosis, Nat. Rev. Cardiol. 6 (2009) 399-409.

[8] A. Tedgui, Z. Mallat, Cytokines in atherosclerosis: pathogenic and regulatory pathways, Physiol. Rev. 86 (2006) 515-581.

[9] V. Parisi, D. Leosco, G. Ferro, A. Bevilacqua, G. Pagano, C. de Lucia, P. PerroneFilardi, A. Caruso, G. Rengo, N. Ferrara, The lipid theory in the pathogenesis of calcific aortic stenosis, Nutr. Metab. Cardiovasc. Dis. 25 (2015) 519-525.

[10] S. Saito, T. Fujiwara, T. Matsunaga, K. Minagawa, K. Fukui, I. Fukuda, T. Osanai, K. Okumura, Increased adiponectin synthesis in the visceral adipose tissue in men with coronary artery disease treated with pravastatin: a role of the attenuation of oxidative stress, Atherosclerosis 199 (2008) 378-383.

[11] K. Łabuzek, Ł. Bułdak, A. Duława-Bułdak, A. Bielecka, R. Krysiak, A. Madej, B. Okopień, Atorvastatin and fenofibric acid differentially affect the release of adipokines in the visceral and subcutaneous cultures of adipocytes that were obtained from patients with and without mixed dyslipidemia, Pharmacol. Rep. 63 (2011) 1124-1136.

[12] T. Mazurek, L. Zhang, A. Zalewski, J.D. Mannion, J.T. Diehl, H. Arafat, L. Sarov-Blat, S. O'Brien, E.A. Keiper, A.G. Johnson, J. Martin, B.J. Goldstein, Y. Shi, Human epicardial adipose tissue is a source of inflammatory mediators, Circulation 108 (2003) 2460-2466.

[13] A.R. Baker, N.F. Silva, D.W. Quinn, A.L. Harte, D. Pagano, R.S. Bonser, S. Kumar, P.G. McTernan, Human epicardial adipose tissue expresses a pathogenic profile of adipocytokines in patients with cardiovascular disease, Cardiovasc. Diabetol. 5 (2006) 1.

[14] V. Parisi, G. Rengo, G. Pagano, V. D'Esposito, F. Passaretti, A. Caruso, M.G. Grimaldi, T. Lonobile, F. Baldascino, A. De Bellis, P. Formisano, N. Ferrara, D. Leosco, Epicardial adipose tissue has an increased thickness and is a source of inflammatory mediators in patients with calcific aortic stenosis, Int. J. Cardiol. 186 (2015) 167-169.

[15] H. Baumgartner, V. Falk, J.J. Bax, M. De Bonis, C. Hamm, P.J. Holm, B. Iung, P. Lancellotti, E. Lansac, D.R. Muñoz, R. Rosenhek, J. Sjögren, P. Tornos Mas, A. Vahanian, T. Walther, O. Wendler, S. Windecker, J.L. Zamorano, ESC Scientific Document Group, 2017 ESC/EACTS guidelines for the management of valvular heart disease, Eur. Heart J. 38 (2017) 2739-2791.
[16] P. Royston, G. Ambler, W. Sauerbrei, The use of fractional polynomials to model continuous risk variables in epidemiology, Int. J. Epidemiol. 28 (1999) 964-974.

[17] A.F. Shorrocks, Decomposition procedures for distributional analysis: a unified framework based on the Shapley value, J. Econ. Inequal. 11 (2013) 99-126.

[18] P. Royston, W. Sauerbrei, Multivariate Model Building. A Pragmatic Approach to Regression Analysis Based On Fractional Polynomials for Modeling Continuous Variables, Wiley, Chichester. UK, 2008 183-186.

[19] M.H. Kutner, C.J. Nachtsheim, J. Neter, W. Li, Applied Linear Statistical Models, $5^{\text {th }}$ edition McGraw Hill, Irwin, New York. NY, 2005409.

[20] A.A. Suleiman, Analysis of multicollinearity in multiple regressions, Int. J. Adv. Technol. Eng. Sci. 3 (2015) 571-578.

[21] G. Iacobellis, M.C. Ribaudo, F. Assael, E. Vecci, C. Tiberti, A. Zappaterreno, U. Di Mario F. Leonetti, Echocardiographic epicardial adipose tissue is related to anthropometric and clinical parameters of metabolic syndrome: a new indicator of cardiovascular risk, J. Clin. Endocrinol. Metab. 88 (2003) 5163e8.

[22] G. Iacobellis, F. Leonetti, Epicardial adipose tissue and insulin resistance in obese subjects, J. Clin. Endocrinol. Metab. 90 (2005) 6300-6302.

[23] S.G. Ahn, H.S. Lim, D.Y. Joe, S.J. Kang, B.J. Choi, S.Y. Choi, M.H. Yoon, G.S. Hwang, S.J. Tahk, J.H. Shin, Relationship of epicardial adipose tissue by echocardiography to coronary artery disease, Heart 94 (2008) e7.

[24] V. Parisi, G. Rengo, P. Perrone-Filardi, G. Pagano, G.D. Femminella, S. Paolillo, L. Petraglia, G. Gambino, A. Caruso, M.G. Grimaldi, F. Baldascino, M. Nolano, A. Elia, A. Cannavo, A. De Bellis, E. Coscioni, T. Pellegrino, A. Cuocolo, N. Ferrara, D. Leosco, Increased epicardial adipose tissue volume correlates with cardiac sympathetic denervation in patients with heart failure, Circ. Res. 118 (2016) 1244-1253.

[25] J.H. Park, Y.S. Park, Y.J. Kim, I.S. Lee, J.H. Kim, J.H. Lee, S.W. Choi, J.O. Jeong, I.W. Seong, Effects of statins on the epicardial fat thickness in patients with coronary artery stenosis underwent percutaneous coronary intervention: comparison of atorvastatin with simvastatin/ezetimibe, J. Cardiovasc. Ultrasound 18 (2010) 121-126.

[26] F. Soucek, N. Covassin, P. Singh, L. Ruzek, T. Kara, M. Suleiman, A. Lerman, C. Koestler, P.A. Friedman, F. Lopez-Jimenez, V.K. Somers, Effects of atorvastatin ( $80 \mathrm{mg})$ therapy on quantity of epicardial adipose tissue in patients undergoing pulmonary vein isolation for atrial fibrillation, Am. J. Cardiol. 116 (2015) 1443-1446.

[27] N. Alexopoulos, B.H. Melek, C.D. Arepalli, G.R. Hartlage, Z. Chen, S. Kim, A.E. Stillman, P. Raggi, Effect of intensive versus moderate lipid-lowering therapy on epicardial adipose tissue in hyperlipidemic post-menopausal women: a substudy of the BELLES trial (beyond endorsed lipid lowering with EBT scanning), J. Am. Coll. Cardiol. 61 (2013) 1956-1961.

[28] M. Abe, M. Matsuda, H. Kobayashi, Y. Miyata, Y. Nakayama, R. Komuro, A. Fukuhara, I. Shimomura, Effects of statins on adipose tissue inflammation: their inhibitory effect on MyD88-independent IRF3/IFN-beta pathway in macrophages, Arterioscler. Thromb. Vasc. Biol. 28 (2008) 871-877.

[29] J. Hartman, W.H. Frishman, Inflammation and atherosclerosis: a review of the role of interleukin- 6 in the development of atherosclerosis and the potential for targeted drug therapy, Cardiol. Rev. 22 (2014) 147-151.

[30] D.F. Gualtero, S.M. Viafara-Garcia, S.J. Morantes, D.M. Buitrago, O.A. Gonzalez, G.I. Lafaurie, Rosuvastatin inhibits interleukin (IL)-8 and IL-6 production in human coronary artery endothelial cells stimulated with Aggregatibacter actinomycetemcomitans serotype b, J. Periodontol. 88 (2017) 225-235.

[31] A. Blyme, C. Asferg, O.W. Nielsen, T. Sehestedt, Y.A. Kesäniemi, C. Gohlke-Bärwolf, K. Boman, R. Willenheimer, S. Ray, C.A. Nienaber, A. Rossebø, K. Wachtell, M.H. Olsen, High sensitivity $C$ reactive protein as a prognostic marker in patients with mild moderate aortic valve stenosis during lipid-lowering treatment: an SEAS substudy, Open Heart 2 (2015), e000152.

[32] P.M. Ridker, From C-Reactive Protein to Interleukin-6 to Interleukin-1: moving upstream to identify novel targets for atheroprotection, Circ. Res. 118 (2016) $145-156$.

[33] G. Iacobellis, H.J. Willens, Echocardiographic epicardial fat: a review of research and clinical applications, J. Am. Soc. Echocardiogr. 22 (2009) 1311-1319.

[34] G. Iacobellis, H.J. Willens, G. Barbaro, A.M. Sharma, Threshold values of high-risk echocardiographic epicardial fat thickness, Obesity 16 (2008) 887-892. 